\title{
O COMPUTADOR NO ENSINO DE ENFERMAGEM - ANÁLISE DAS ATITUDES DE DISCENTES DE INSTITUIÇÕES DE NÍVEL SUPERIOR
}

Paulo Celso Prado Telles Filho*

Silvia Helena De Bortoli Cassiani**

FILHO, P.C.P.T.; CASSIANI, S.H.de B. O computador no ensino de enfermagem - análise das atitudes de discentes de instituições de nível superior. Rev.latino-am.enfermagem, Ribeirão Preto, v. 7, n. 1, p. 93-98, janeiro 1999.

Este estudo teve por objetivo identificar e analisar atitudes de discentes de instituições de ensino superior sobre utilização do computador no ensino de enfermagem. A metodologia consistiu na elaboração, aplicação e análise de escalas de Likert distribuidas entre discentes do oitavo semestre de instituições de ensino de Ribeirão Preto e Bauru. Os resultados indicam o computador como um dos principais recursos para o ensino, a simulação computadorizada como excelente ponte entre teoria e prática e os softwares educacionais úteis e interessantes. Verificou-se elevado nível de interesse e atitudes positivas em relação ao emprego do recurso computacional no ensino de enfermagem.

UNITERMOS: enfermagem, ensino, computação

\section{INTRODUÇÃO}

A informática em saúde é definida como a área do saber que trata da aplicação e utilização das ferramentas de automação de processamento de dados nos vários segmentos de atividades relacionadas à saúde do indivíduo e da coletividade BRASIL (1988).

Destacamos, no entanto, a utilização do computador no ensino de enfermagem. As primeiras experiências no que diz respeito ao ensino de enfermagem ligado à computação foram iniciadas durante a década de sessenta nos Estados Unidos. Em nosso país estas experiências se deram apenas na metade da década de oitenta, em instituições de ensino superior.

As funções mais comuns do computador são: controle do ambiente educacional (uma vez que o computador é capaz de gerar relatórios que analisam a atuação do estudante e a efetividade do programa de enfermagem), avaliação (necessária para que o docente possa avaliar, intervir e guiar o estudante) e instrução (o mais promissor uso do computador na enfermagem), podendo oferecer características instrucionais únicas SANTOS et al. (1993).

Na modalidade Simulação, o computador apresenta ao educando uma situação real para ser trabalhada ou resolvida o que o torna um instrumento gerador de descobertas e informações.

As simulações clínicas computadorizadas mostram-se também extremamente úteis ao processo de enfermagem em praticamente todas as áreas de atendimento (BERSKY, 1995).

Existem dois tipos de simulação: estática (na qual a representação dos modelos é fixa e o educando pode interagir somente com um pequeno número de variáveis pré determinadas) e dinâmica (aquela em que o educando intervém ativamente, podendo adicionar ou retirar variáveis).

Estudos recentes apontam a simulação computadorizada como algo de indescritível proveito. As etapas a serem consideradas para sua construção são aqui citadas: seleção da situação problema, esboço do problema, desenvolvimento e revisão.

Segue-se algumas das importantes vantagens da utilização da simulação computadorizada: proporciona a aprendizagem pela descoberta, oportuniza o desenvolvimento de habilidades para solução de problemas, torna o ensino uma experiência ativa, faz a ligação entre conhecimento teórico e aplicação prática, seleciona e testa hipóteses desenvolvendo habilidade de tomar decisões, desenvolve o raciocínio lógico e proporciona o desenvolvimento da criatividade.

No ensino tradicional é impossível oferecer, a

* Discente do $7^{\circ}$ semestre do Curso de Graduação da Escola de Enfermagem de Ribeirão Preto da Universidade de São Paulo. Bolsista do Programa Especial de Treinamento - CAPES/EERP-USP

* Professora Doutora do Departamento de Enfermagem Geral e Especializada da Escola de Enfermagem de Ribeirão Preto da Universidade de São Paulo e Tutora do Programa Especial de Treinamento - CAPES/EERP-USP 
cada estudante, a mesma experiência de trabalho real e manuseio prático, o que pode ser obtido através da instrução por computador, que proporciona um ensino individualizado, respeitando o ritmo próprio e a disponibilidade de cada educando.

SANTAROSA (1985) afirma que o computador na educação é um meio para o ensino de áreas de conteúdo diversificado e para auxílio do professor em sua tarefa de ensinar. Como exemplo de sistema de ensino temos Computer Assisted Instruction - CAI (Instrução Auxiliada Por Computador) na qual as experiências de aprendizagem são previamente estruturadas para diferentes tipos de educandos. O sistema tem se mostrado mais efetivo que a instrução por métodos tradicionais pois, no aprendizado individualizado, o aprendiz controla o ritmo de sua própria instrução.

CAI refere-se a qualquer técnica que se apoie em um computador para facilitar a aprendizagem e se subdivide em dois ramos: Instrução CONTROLADA por Computador e Instrução ASSISTIDA por Computador. A primeira refere-se ao armazenamento de informações sobre recursos disponíveis de aprendizagem que foram integrados para oferecer programas de instrução para uso individual e para facilitar o gerenciamento de recursos operacionais, podendo ser programado para testar conhecimento, analisar respostas de educandos e armazenar informações. A segunda proporciona exatidão aumentada e maior acesso à informação atualizada, o que representa vantagens financeiras e de economia de tempo.

A utilização do computador no controle da educação é comumente chamada de EDUCAÇÃO CONTROLADA PELO COMPUTADOR (ECC), que constata a superioridade do desempenho do aluno na disciplina quando relacionando-se alunos que interagiram com os microcomputadores em contraste com aqueles não expostos a esse meio.

O computador no ensino de enfermagem é de fundamental importância. ADAMS (1986) afirma que populações futuras serão compostas por alfabetizados e analfabetos em computação e recomenda que profissões que desejem conviver com outras esferas da sociedade devam alfabetizar-se.

\section{OBJETIVOS}

Este estudo tem como objetivos:

Identificar e analisar as atitudes dos discentes de duas instituições de ensino superior em enfermagem sobre a utilização do computador no ensino de enfermagem.

Determinar e comparar as respostas obtidas entre os discentes das citadas instituições.

\section{METODOLOGIA}

\subsection{População em estudo}

A população em estudo consta de discentes cursando o oitavo semestre das seguintes instituições de ensino superior: A (Universidade Estadual) e B (Universidade Particular), localizadas respectivamente nas cidades de Ribeirão Preto e Bauru. A amostra se constituiu daqueles que responderam aos instrumentos de coleta de dados, ou seja: 101 discentes.

Faz-se necessário destacar que a escolha das universidades estadual e particular deveu-se ao interesse de analisar universos com linhas pedagógicas, alunado, direcionamento e obtenção de recursos financeiros diferentes. A escolha do oitavo semestre deu-se ao fato de optar-se por coletar informações de discentes em término do Curso de Graduação, tendo já passado por várias etapas dos processos que lhes foram proporcionados, dispondo de maiores conhecimentos e experiências.

\subsection{Instrumento de coleta de dados}

Como instrumento de coleta de dados desenvolveu-se e utilizou-se dez escalas de Likert, que constituem de uma série de itens favoráveis e desfavoráveis ao objeto de estudo, aos quais solicita-se ao discente uma resposta. A cada resposta é atribuído um valor numérico que indica o nível de concordância, indecisão ou discordância em relação ao que lhe é afirmado.

Utilizamos esta escala contendo três pontos, variando numericamente de um a três.

Para a construção das escalas foram utilizados os seguintes procedimentos:

$1^{\circ}$ Reunião de um "pool" de frases com base na experiência dos investigadores e literatura consultada.

$2^{\circ}$ Seleção de frases indicativas e pertinentes à temática.

$3^{\circ}$ Seqüência de frases, seguindo grau de complexidade.

$4^{\circ}$ Realização de estudo piloto com seis juízes.

$\mathrm{O}$ estudo piloto visou verificar a pertinência do instrumento, avaliar validade de conteúdo e analisar dados da escala. Após a análise do resultado do estudo piloto, o instrumento foi modificado e aplicado na população descrita.

Apresentamos abaixo um exemplo de escala de Likert utilizada: 
Item 1: Considero o computador um dos principais recursos para o ensino de enfermagem.

\begin{tabular}{|c|c|c|}
\hline 1 & 2 & 3 \\
\hline Desaprovo & Indeciso & Aprovo \\
\hline
\end{tabular}

\subsection{Análise dos dados}

A análise dos dados deu-se através dos programas EXCEL (planilhas para o cômputo dos dados) e do programa MANAP (freqüências de respostas).

\section{RESULTADOS E DISCUSSÃO}

Anteriormente à apresentação e análise das tabelas, faz-se necessário o esclarecimento sobre a não ocorrência de diferença significativa entre as atitudes dos discentes das universidades analisadas, motivo pelo qual a análise deu-se baseada em colunas (análise vertical) e razão também pela qual as letras que acompanham os números são sempre a (as minúsculas). Para o limite entre significância e não significância adotou-se o valor 0,05 . A população analisada foi composta por todos os alunos do oitavo semestre das instituições analisadas, sendo 68 oriundos da instituição A e 33 da instituição B. Obtevese devolução da totalidade dos instrumentos de coleta de dados aplicados.

A seguir apresentam-se, separadamente, os dez itens e tabelas das citadas escalas: com os respectivos graus (desaprovação, indecisão, aprovação), considerando as Universidades A e B. Posteriormente faz-se uma análise quantitativa dos resultados bem como um breve comentário acerca da temática.

Item 1: Considero o computador um dos principais recursos para o ensino de enfermagem.

\begin{tabular}{c|c|c|c|c}
\hline Universidade & Desaprovo & Indeciso & Aprovo & Total \\
\hline A & $0,162 \mathrm{a}$ & $0,265 \mathrm{a}$ & $0,574 \mathrm{a}$ & 68 \\
$\mathrm{~B}$ & $0,273 \mathrm{a}$ & $0,121 \mathrm{a}$ & $0,606 \mathrm{a}$ & 33 \\
\hline & $\mathrm{p}>0,05$ & $\mathrm{p}>0,05$ & $\mathrm{p}>0,05$ & 101 \\
\hline
\end{tabular}

Verifica-se através desta tabela o aumento gradativo em relação à aprovação ao item, ou seja, 20 desaprovações, 22 indecisões e 59 aprovações. Os dados aqui verificados estão em concordância com a literatura, que denota elevado índice de aprovação da utilização do computador em diversas áreas da enfermagem, principalmente em relação ao ensino.
Item 2: Acredito que alunos que interagem com microcomputadores apresentam igual ou menor desempenho em relação àqueles não expostos a esse meio.

\begin{tabular}{c|c|c|c|c}
\hline Graus & Desaprovo & Indeciso & Aprovo & Total \\
Universidade & & & & \\
\hline A & $0,691 \mathrm{a}$ & $0,191 \mathrm{a}$ & $0,118 \mathrm{a}$ & 68 \\
$\mathrm{~B}$ & $0,515 \mathrm{a}$ & $0,273 \mathrm{a}$ & $0,212 \mathrm{a}$ & 33 \\
\hline & $\mathrm{p}>0,05$ & $\mathrm{p}>0,05$ & $\mathrm{p}>0,05$ & 101 \\
\hline
\end{tabular}

Nota-se o elevado índice de desaprovação a este item, contando com 64 respostas de desaprovação, seguindo-se de 22 indecisões e 15 aprovações.

O fato da utilização dos microcomputadores relacionada a elevado desempenho, no processo ensinoaprendizado é aprovado pelos discentes, o que o denota conhecimento e atualização em relação ao processo de informatização que ocorre nos mais diversos setores.

Item 3: Acredito que a instrução por computador ofereça um ensino individualizado, que respeite o ritmo próprio e a disponibilidade de cada educando.

\begin{tabular}{c|c|c|c|c}
\hline Graus & Desaprovo & Indeciso & Aprovo & Total \\
Universidade & & & & \\
\hline A & $0,103 \mathrm{a}$ & $0,103 \mathrm{a}$ & $0,794 \mathrm{a}$ & 68 \\
$\mathrm{~B}$ & $0,182 \mathrm{a}$ & $0,182 \mathrm{a}$ & $0,636 \mathrm{a}$ & 33 \\
\hline & $\mathrm{p}>0,05$ & $\mathrm{p}>0,05$ & $\mathrm{p}>0,05$ & 101 \\
\hline
\end{tabular}

Percebe-se uma das mais elevadas freqüências de aprovação neste item: 75, sendo seguido igualmente de 13 indecisões e 13 desaprovações.

É crescente a quantidade de indivíduos, das diversas áreas do ensino a valorizar a instrução através do computador. Atualmente existem vários estudos a comprovar a eficácia deste novo instrumento de ensino.

Item 4: A simulação computadorizada não faz a ligação entre teoria e prática.

\begin{tabular}{c|c|c|c|c}
\hline Universidade & Desaprovo & Indeciso & Aprovo & Total \\
\hline A & $0,721 \mathrm{a}$ & $0,191 \mathrm{a}$ & $0,088 \mathrm{a}$ & 68 \\
$\mathrm{~B}$ & $0,667 \mathrm{a}$ & $0,091 \mathrm{a}$ & $0,242 \mathrm{a}$ & 33 \\
\hline & $\mathrm{p}>0,05$ & $\mathrm{p}>0,05$ & $\mathrm{p}>0,05$ & 101 \\
\hline
\end{tabular}

Quanto a este item, verifica-se claramente o elevado grau de desaprovação: 71 indivíduos, seguindo de 16 indecisos e 14 aprovações. 
A simulação computadorizada é dentre os benefícios advindos da utilização do computador no ensino de Enfermagem um dos mais úteis, visto ser o responsável pela maior facilidade de desenvolvimento no que se refere à tomada de decisão, principalmente em situações práticas de estágio.

Item 5: Acho estimulante e prazerosa a utilização do computador.

\begin{tabular}{c|c|c|c|c}
\hline Universidade & Desaprovo & Indeciso & Aprovo & Total \\
\hline A & $0,088 \mathrm{a}$ & $0,088 \mathrm{a}$ & $0,824 \mathrm{a}$ & 68 \\
B & $0,059 \mathrm{a}$ & $0,176 \mathrm{a}$ & $0,765 \mathrm{a}$ & 33 \\
\hline & $\mathrm{p}>0,05$ & $\mathrm{p}>0,05$ & $\mathrm{p}>0,05$ & 101 \\
\hline
\end{tabular}

Registra-se neste item o mais elevado índice de aprovação das escalas desta pesquisa: 82 aprovações. Ainda neste item, o menor número de indecisões: 11 discentes e 8 desaprovações.

Interessante notar a visualização do computador não só como um instrumento de obtenção de melhores resultados, mas sim instrumento de estímulo e prazer.

Item 6: A instrução tradicional obtém melhores resultados que a instrução através do computador.

\begin{tabular}{c|c|c|c|c}
\hline Graus & Desaprovo & Indeciso & Aprovo & Total \\
Universidade & & & & \\
\hline $\mathrm{A}$ & $0,471 \mathrm{a}$ & $0,397 \mathrm{a}$ & $0,132 \mathrm{a}$ & 68 \\
$\mathrm{~B}$ & $0,394 \mathrm{a}$ & $0,333 \mathrm{a}$ & $0,242 \mathrm{a}$ & 33 \\
\hline & $\mathrm{p}>0,05$ & $\mathrm{p}>0,05$ & $\mathrm{p}>0,05$ & 101 \\
\hline
\end{tabular}

Com 45 desaprovações, 38 indecisões e 18 aprovações, apresentam-se os resultados desta tabela, através da qual observa-se claramente a busca por novos métodos de instrução e técnicas pedagógicas. Destacase, neste item um elevado índice de indecisão, fato que pode ser explicado pelo pouco contato dos discentes com a instrução através do computador, devido ao baixo emprego e até mesmo à inexistência destes recursos no ensino superior ou mesmo pela real indecisão entre o método tradicional e a instrução através do computador.

Item 7: O computador não é muito utilizado pelos discentes de nível superior.

\begin{tabular}{c|c|c|c|c}
\hline Graus & Desaprovo & Indeciso & Aprovo & Total \\
Universidade & & & & \\
\hline A & $0,662 \mathrm{a}$ & $0,176 \mathrm{a}$ & $0,162 \mathrm{a}$ & 68 \\
B & $0,455 \mathrm{a}$ & $0,303 \mathrm{a}$ & $0,242 \mathrm{a}$ & 33 \\
\hline & $\mathrm{p}>0,05$ & $\mathrm{p}>0,05$ & $\mathrm{p}>0,05$ & 101 \\
\hline
\end{tabular}

Verifica-se a desaprovação de 60 discentes, a qual se segue de 22 indecisões e 19 aprovações. Através destes resultados constata-se o elevado nível da compreensão e entendimento da importância do computador e sua conseqüente utilização.

Item 8: Os programas de computação (Excel, Windows, Power Point, Corel Draw, etc) não facilitam mas sim dificultam a "vida acadêmica".

\begin{tabular}{c|c|c|c|c}
\hline Graus & Desaprovo & Indeciso & Aprovo & Total \\
\hline Unidade & & & & \\
\hline B & $0,897 \mathrm{a}$ & $0,059 \mathrm{a}$ & $0,044 \mathrm{a}$ & 68 \\
& $0,515 \mathrm{a}$ & $0,394 \mathrm{a}$ & $0,091 \mathrm{a}$ & 33 \\
\hline & $\mathrm{p}>0,05$ & $\mathrm{p}>0,05$ & $\mathrm{p}>0,05$ & 101 \\
\hline
\end{tabular}

Destaca-se o maior índice de desaprovação dentre todos os itens: 78 discentes, o que aponta para a consciência da facilidade proporcionada pelos diversos programas de computação. Neste item registram-se também 6 aprovações à afirmação (a mais baixa de todos os itens) e 17 indecisões.

Item 9: Acredito que o computador proporcione o desenvolvimento do raciocínio lógico e da criatividade.

\begin{tabular}{c|c|c|c|c}
\hline Universidade & Desaprovo & Indeciso & Aprovo & Total \\
\hline A & $0,029 \mathrm{a}$ & $0,162 \mathrm{a}$ & $0,809 \mathrm{a}$ & 68 \\
B & $0,061 \mathrm{a}$ & $0,242 \mathrm{a}$ & $0,697 \mathrm{a}$ & 33 \\
\hline & $\mathrm{p}>0,05$ & $\mathrm{p}>0,05$ & $\mathrm{p}>0,05$ & 101 \\
\hline
\end{tabular}

Após a leitura da tabela, observa-se 78 aprovações, 19 indecisões e 4 desaprovações (o menor índice), subentendendo-se, o computador enquanto instrumento aliado à criatividade e ao raciocínio lógico.

Item 10: Os softwares educacionais são extremamente monótonos e não apresentam vantagens.

\begin{tabular}{c|c|c|c|c}
\hline Graus & Desaprovo & Indeciso & Aprovo & Total \\
Universidade & & & & \\
\hline A & $0,632 \mathrm{a}$ & $0,324 \mathrm{a}$ & $0,044 \mathrm{a}$ & 68 \\
$\mathrm{~B}$ & $0,515 \mathrm{a}$ & $0,333 \mathrm{a}$ & $0,152 \mathrm{a}$ & 33 \\
\hline & $\mathrm{p}>0,05$ & $\mathrm{p}>0,05$ & $\mathrm{p}>0,05$ & 101 \\
\hline
\end{tabular}

Observa-se 60 desaprovações, 33 indecisões e 8 aprovações em relação à presente afirmação. Destacase a quantidade de indivíduos que percebem os softwares educacionais enquanto interessantes e vantajosos, o que demonstra atualização de conhecimentos sobre os 
mesmos. Atualmente é crescente a produção e qualidade de softwares nas mais diversas áreas de ensino.

\section{CONCLUSÕES}

O conhecimento científico e tecnológico é atualmente um processo em acelerado desenvolvimento. O computador como principal instrumento da informática auxilia na organização do elevado volume de informações da atual sociedade.

Segundo SANTOS et al. (1981) são inúmeras e evidentes as possibilidades da utilização deste instrumento no ensino de enfermagem. Educadores e educandos desta área necessitam estar familiarizados com computadores para poder obter deste novo recurso tecnológico máximas vantagens.

Face ao exposto faz-se interessante a identificação das atitudes de discentes universitários acerca da utilização dos recursos computacionais no auxílio do ensino em enfermagem.

Primeiramente faz-se necessário esclarecer que não houve diferença significativa entre as instituições analisadas (o que foi demonstrado pelo $\mathrm{p}$ value maior que $0,05)$ e que o fato da existência de valores numéricos diferentes entre as instituições A e B não implica em diferença estatisticamente significante.

Os resultados que ora se apresentam levam em consideração as duas instituições analisadas e estão em porcentagem para proporcionar maior facilidade de entendimento: $58,4 \%$ consideram o computador um dos principais recursos para o ensino de enfermagem; $63,3 \%$ acreditam que discentes que interagem com microcomputadores apresentam melhor desempenho em relação a outros; $74,2 \%$ acreditam que a instrução por computador ofereça ensino individualizado, que respeita o ritmo próprio e a disponibilidade do educando; 70,2\% concebem a simulação como "ligação" entre teoria e prática e $81,1 \%$ acham estimulante e prazerosa a utilização do computador. A instrução tradicional obtém melhores resultados que através do computador segundo $17,8 \% ; 59,4 \%$ relatam o computador como instrumento utilizado por discentes de nível superior; $77,2 \%$ percebem os programas computacionais, tais como Windows, Corel Draw, Power Point e Excel como facilitadores das tarefas acadêmicas; $77,2 \%$ acreditam que o computador proporcione o desenvolvimento do raciocínio lógico e da criatividade e $59,4 \%$ afirmam serem os softwares educacionais interessantes e vantajosos.

É importante destacar o item $\mathrm{n}^{\circ}$ 5: Acho estimulante e prazerosa a utilização do computador, como sendo aquele que obteve maior grau de aprovação, ou seja $81 \%$.

Este estudo detectou atitudes extremamente positivas dos indivíduos analisados frente a utilização do recurso computacional no ensino de enfermagem, bem como atualização no que diz respeito a novas técnicas pedagógicas, interesse e prazer advindos da utilização do recurso computacional.

Comprova-se a localização dos referidos discentes na terceira fase da educação, já que, como refere CIETTO (1986): "Historicamente, podemos identificar três grandes fases na educação: a era que precedeu a invenção da imprensa, a era pós Gutemberg e, finalmente, a era do ensino assistido por computador.

Agradecimento: Agradeço ao Prof.Dr. Carlos Roberto Padovani do Instituto de Biociências do Departamento de Bioestatística da UNESP-Campus de Botucatu pela contribuição referente aos procedimentos de estatística

\section{THE COMPUTER IN THE TEACHING OF NURSING - ANALYSYS OF THE ATTITUDES BY STUDENTS FROM UNIVERSITY INSTITUTIONS}

This study aims at identifying and analyzing the attitudes by students from different institutions regarding the use of the computer in Nursing teaching. Methodology consisted in the elaboration, application and analysis of Likert scales distributed among students of the eighth semester in higher education institutions at the cities of Ribeirão Preto and Bauru. Results indicated the computer as one of the main resources for nursing teaching and the simulation as an excellent bridge between theory and practice as well as the educational softwares useful and interesting. Authors verified the high level of interest and positive attitudes regarding the employment of computer resources in nursing teaching.

KEY WORDS: nursing, teaching, computer

\section{EL COMPUTADOR EN LA ENSEÑANZA DE ENFEMERÍA - ANÁLISIS DE LAS ACTITUDES DE DISCENTES DE INSTITUICIONES DE NIVEL SUPERIOR}


la utilización del computador en la enseñanza de enfermería. La metodología consistió en la elaboración, aplicación y análisis de escalas tipo Likert distribuidas entre discentes de octavo semestre de instituciones de enseñanza de Ribeirão Preto y Bauru. Los resultados indican que el computador es uno de los principales recursos para la enseñanza y la simulación computadorizada es un excelente puente entre teoría y práctica, los softwares educacionales son útiles e interesantes. Se verificó elevado nivel de interés y actitudes positivas en relación al empleo del recurso computacional en la enseñanza de enfermería.

TÉRMINOS CLAVES: enfermería, enseñanza, computación

\section{REFERÊNCIAS BIBLIOGRÁFICAS}

01. ADAMS, G.A. Computer technology: its impact on nursing practice. Nursing Administration Quartely. v.10, n.2, p.21-33, 1986.

02. BERSKY, A.K. Building a nursing activity database for processing free-text entry during computerized clinical simulation testing. Computers in Nursing, v. 13, n.5, p.236-243, sept/oct, 1995.

03. BRASIL. Proposta de plano setorial de informática em saúde. Relatório da comissão especial de informática em saúde. Secretaria Especial de Informática em Saúde. Brasília, 1988

04. CieTtO, L. O impacto da informática na enfermagem. Revista Brasileira de Informática em Saúde, São Paulo, v.1, n.1, p.1519, set/out. 1986.
05. SANTOS, B.R.L.; FUNCKE, L.B.; RIBEIRO, N.R.R.; UEBEL, W.S. Percepção dos alunos de graduação sobre a informática no ensino de enfermagem. Rev.Gaúch.Enfermagem, Porto Alegre, v.14, n.1, p.40-44, jan. 1993.

06. SANTOS, B.R.L.; SCOCHI, C.G.S.; ÉVORA, Y.D.M. O computador como instrumento de apoio na pesquisa e ensino de enfermagem. XXXIX Congresso Brasileiro de Enfermagem, Salvador, Ba. nov. 1981.

07. SANTAROSA, L.M.C. Análise e perspectiva da utilização do computador como recurso instrucional - Tecnologia Educacional. Rio de Janeiro. v. 32, n.1, p.16-26, jan./fev. 1985. 